\title{
Bending-Induced Conductance Increase in Individual Semiconductor Nanowires and Nanobelts
}

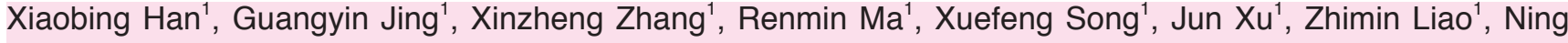 \\ Wang $^{2}$, and Dapeng $\mathrm{Yu}^{1}(\bowtie)$ \\ ${ }^{1}$ State Key Laboratory for Mesoscopic Physics, and Electron Microscopy Laboratory, Department of Physics, Peking University, \\ Beijing 100871, China \\ ${ }^{2}$ Physics Department, Hong Kong University of Science and Technology, Hong Kong, China \\ Received: 9 March 2009 / Revised: 15 April 2009 / Accepted: 2 May 2009 \\ (C)Tsinghua University Press and Springer-Verlag 2009. This article is published with open access at Springerlink.com
}

\begin{abstract}
Reliable ohmic contacts were established in order to study the strain sensitivity of nanowires and nanobelts. Significant conductance increases of up to $113 \%$ were observed on bending individual $\mathrm{ZnO}$ nanowires or CdS nanobelts. This bending strain-induced conductance enhancement was confirmed by a variety of bending measurements, such as using different manipulating tips (silicon, glass or tungsten) to bend the nanowires or nanobelts, and is explained by bending-induced effective tensile strain based on the principle of the piezoresistance effect.
\end{abstract}

\section{KEYWORDS}

$\mathrm{ZnO}$ nanowires, bending strain, piezoresistance, conductance enhancement.

Semiconductor nanowires (NWs) and nanobelts (NBs) are currently attracting considerable interest as they are expected to play an important role in the development of nanodevices and nanotechnologies [1-3]. Recently, the electromechanical properties of nanowires and nanobelts have attracted great attention [4-7]. In particular, piezoelectric $\mathrm{ZnO}$ NW arrays have been utilized successfully as a nanogenerator [8]. Great emphasis has been put on establishing a Schottky contact with the NWs, so that piezoelectric gates and contact potentials can be utilized as sensors and switches $[9,10]$. To study the strain (especially bending strain) effect on the intrinsic electronic transport properties of NWs or
NBs, ohmic contacts are highly desirable, so that the observed conductance change arises mainly from the NW or NB without the influence of the contacts. Here we show that a large increase in conductance can be induced by bending individual $\mathrm{ZnO}$ NWs or CdS NBs with ohmic contacts. Using different manipulating tips (silicon, glass and metal), a variety of bending measurements were conducted in order to confirm the bending modulation of these nanostructures. Our observed phenomena have been explained by the piezoresistance effect.

ZnO NWs were prepared by a simple method of physical vapor deposition [11]. The synthesis was carried out in a conventional furnace with a

Address correspondence to yudp@pku.edu.cn 
horizontal alumina tube, and the growth direction of the ZnO NWs was [001]. The nanowires were dispersed into ethanol and a single $\mathrm{ZnO}$ NW (about $300 \mathrm{~nm}$ in diameter) was transferred over a trench on an $\mathrm{SiO}_{2}$-covered (500 nm) Si substrate by a glass fiber under an optical microscope. A dual beam focused ion beam (DB235-FIB) system was used to etch the trenches and to deposit Pt electrodes in order to pin the $\mathrm{ZnO} \mathrm{NW}$ of interest, as depicted schematically in the insets of Fig. 1 . The linear $I-V$ characteristic of the nanowire indicates that good ohmic contacts have been achieved. The problem of how FIB-deposited $\mathrm{Pt}$ affects our experiments must be considered. The FIB-affected region is about $1 \mu \mathrm{m}$, where $\mathrm{Pt}$ or Ga may injected into the $\mathrm{ZnO}$ nanowires to improve the contact properties, and in an extended region of about $2-3 \mu \mathrm{m}$, a very thin carbon film will be deposited. Outside this extended region, the effect of FIB can be neglected. As our NWs is over $40 \mu \mathrm{m}$

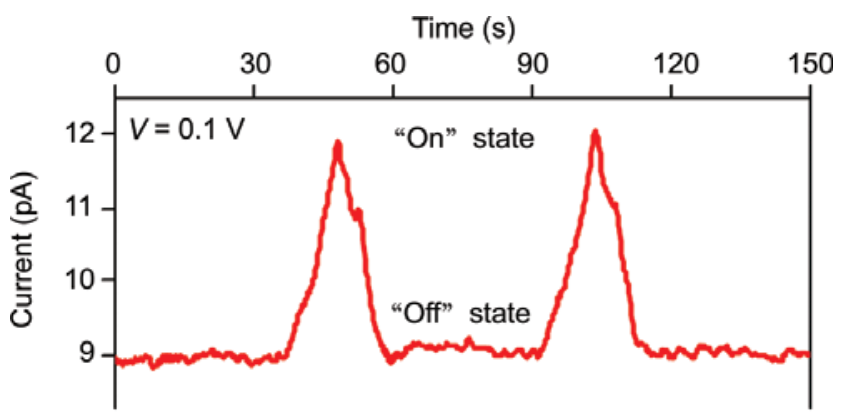

(a)

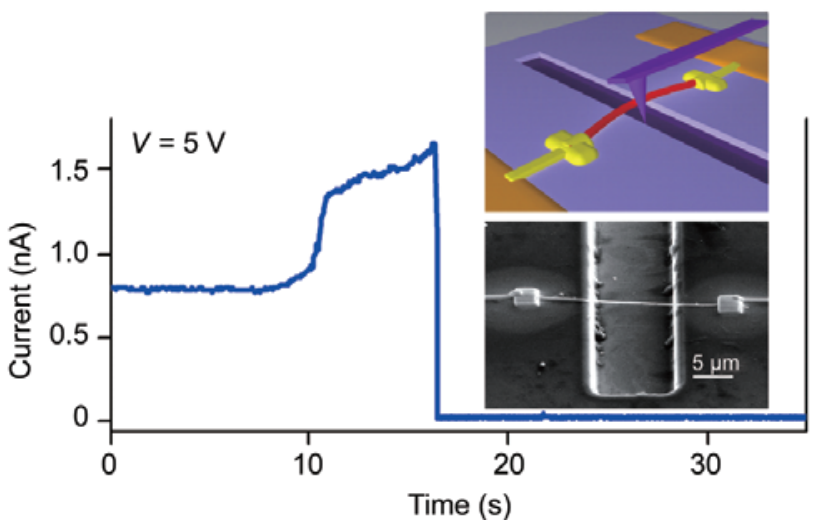

(b)

Figure 1 (a) The current-time relationship with a lateral push along the trench to bend the $\mathrm{ZnO} N W$, where the two peaks identify two conductance cycles of bending and release; (b) the ZnO NW breaks under an increased bending deformation. The bias voltages in (a) and (b) are $0.1 \mathrm{~V}$ and $5 \mathrm{~V}$, respectively. Upper inset: Schematic illustration of the process. Lower inset: SEM image of a single ZnO NW over a trench. The scale bar is $10 \mu \mathrm{m}$ in length and the middle region of about $10-20 \mu \mathrm{m}$ suffers the actual bending strain, we can study the bending strain effect on the $\mathrm{ZnO}$ nanowires while utilizing the stable ohmic contacts. A scanning probe microscope (SPM, 3800N, Seiko) was used for the bending experiments. The dynamic force microscope mode was used for searching and locating the $\mathrm{ZnO}$ NWs during operation, and the AFM mode (silicon AFM tip) was employed to push the nanowires. A small lateral push was applied by moving the AFM tip along the trench; the current increased dramatically with strain and dropped when the strain was released, leaving conductance pulses, as shown in Fig. 1(a). Two current peaks appeared with an inter-peak time of $50 \mathrm{~s}$. Applying a very large lateral bending force led to breaking of the NW and an abrupt drop in the current to zero. The largest increase in conductivity was about $113 \%$, as shown in Fig. 1(b). The low-conductance state can represent the off (" 0 ") state, and the high conductance the on $(" 1$ ") state. Therefore, modulation of the conductance in $\mathrm{ZnO}$ NWs via bending can be used as a nanoscale switch or other electromechanical device in nanoelectromechanical systems (NEMS).

To exclude the possibility that the observed enhanced conductance may be caused by other effects, such as a static charging effect due to the conducting AFM tip, a glass fiber was used to deform the NW under an optical microscope. Figure 2(a) shows the current-time relationship at a constant bias voltage of $0.1 \mathrm{~V}$. Bending the NW to a certain position and holding that bending deformation produced a very clear current step. Due to vibrations in the apparatus (noise on the current stage) the bent NW was broken after less than $20 \mathrm{~s}$. The currenttime curve is consistent with that obtained using a conducting AFM tip. Attention should be paid to where the NW was bent and broken. As we can see from the insets in Fig. 2(a), deformation and breaking happened in the part over the trench. Furthermore, using a tungsten tip instead of a glass tip to conduct similar experiments gave the same result.

Our experiments were also extended to $\mathrm{CdS}$ NBs. As shown in Fig. 2(b), a glass fiber tip was manipulated to a position under the CdS NB with fixed ends. As the tip was tilted and pushed forward, 
the NB was bent. Similar to the experiment for $\mathrm{ZnO}$ NWs, we observed a bending-induced enhancement in conductance, and generated current stairs by modifying the applied bending force. This means that the different materials have a similar response under bending deformation.

Since the stability of the contacts between the NW and the electrodes may affect the $I-V$ results during the bending experiment, a free moving contact was generated in order to conduct static electromechanical measurements. Different from the first two dynamic measurements-in which measuring the $I-V$ curve is impossible while maintaining a stable bending strain deformation-we bent the NW to different degrees, and then statically measured the $I-V$ curves. In this way, we correlate the bending strain $(D / 2 R)$ with the conductance change, where $D$ is the diameter of the

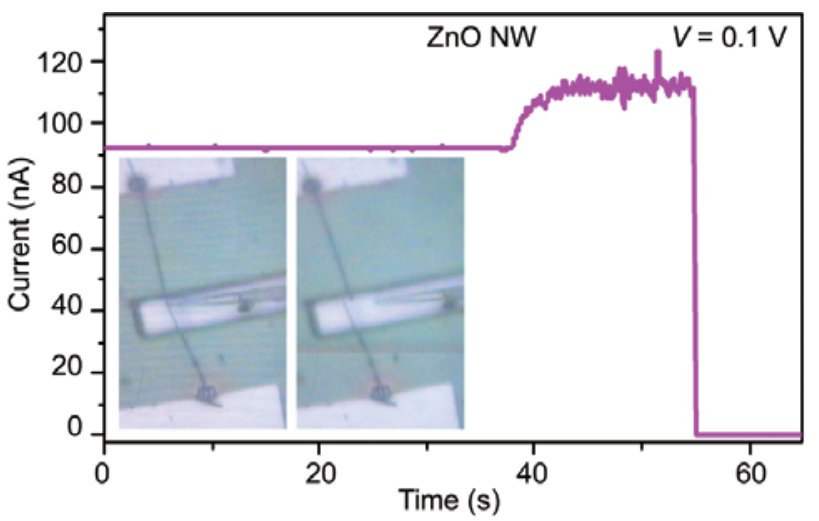

(a)

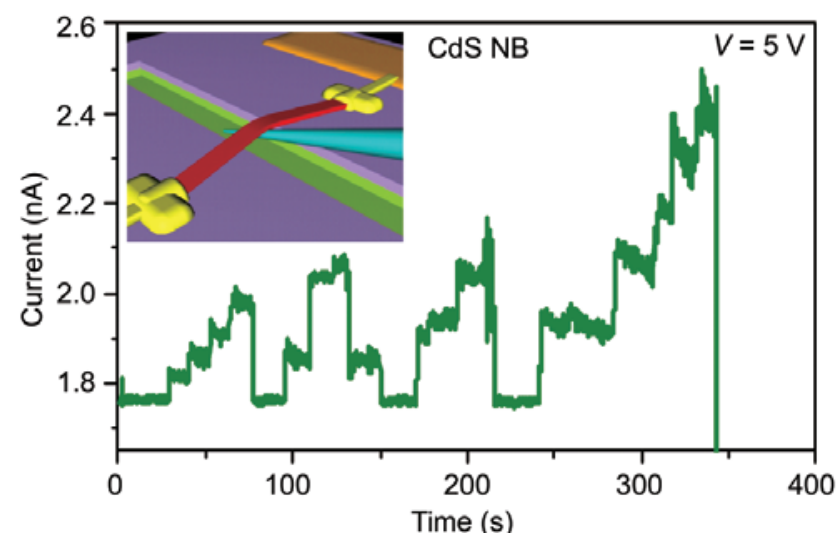

(b)

Figure 2 (a) The current-time relationship (under a bias voltage of $0.1 \mathrm{~V}$ ) using a glass tip to bend $\mathrm{ZnO} \mathrm{NW}$ under an optical microscope. The insets are optical photographs corresponding to holding a bent state (left), and after being broken (right); (b) similar experimental results for CdS NB (under a bias voltage of $5 \mathrm{~V}$ ); stairs appear by modifying the bending force nanowire (400 $\mathrm{nm}$ in this sample) and $R$ is the radius of curvature of the bent nanowire. In this experiment, we deposited Pt on the ends of a $\mathrm{ZnO}$ NW lying on two nearby electrodes. Under the optical microscope, we used a glass fiber to occasionally move one of the NW ends together with the Pt, which was originally deposited on the electrode to fix the nanowire. In this way, we can maintain both metal-to-metal ohmic contact and a free moving end of the NW for the bending experiment. As shown in Fig. 3, as the bending degree increased, the conductance showed a corresponding increase; as the bending was released, the conductance fell accordingly. When the NW end was returned to its original position, with some deformation still present in the NW, the conductance was slightly higher than the original value. Careful observation showed a minor asymmetry of the changes in the two bias regions; in our opinion, this minor asymmetry may arise from the moving contact, which may induce a very low contact barrier. The insert shows the bending strain-conductance relationship - the conductance was increased by as much as $10.7 \%$ when the bending strain reached $0.21 \%$. These linear $I-V$ curves and the reversible modulation further confirm that the contacts in our electromechanical experiments were stable ohmic in nature.

Bending can change the band structure [12] of $\mathrm{ZnO}$ NWs, which will significantly affect the electronic transport properties of individual $\mathrm{ZnO}$ NWs. Especially, this bending-induced inhomogeneous strain (outer tensile and inner compressive) will narrow the band gap and warp the band, resulting in an effective tensile strain along the NW. As the bending strain is inhomogeneous, tensile and compressive strain, shear strain (even in the middle part) coexist, and the bottom of the conduction band will split into broad sub-bands, each representing a different kind of strain effect. The tensile strain and shear strain tend to narrow the band gap, since their states are much lower than the compressive states. The free electron carriers will be mainly distributed in the lower sub-bands; this is why the tensile strain takes effect. Another reason is that the electron effective mass in different crystal directions can be affected differently by this complicated 


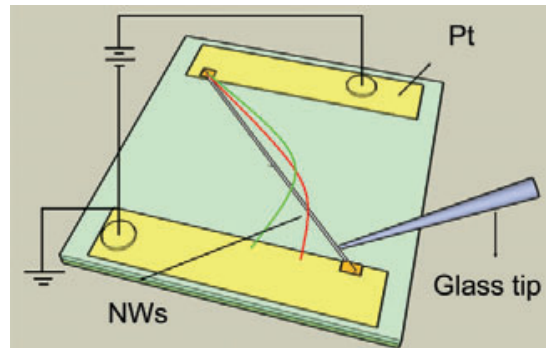

(a)

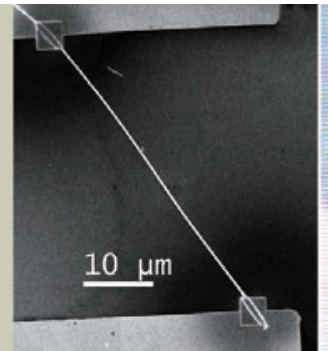

(b)

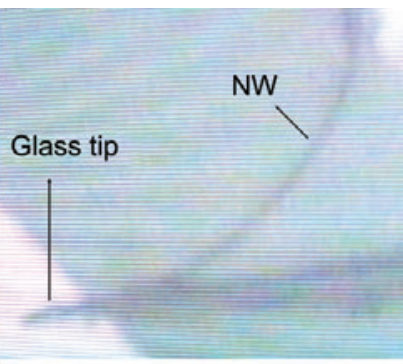

(c)
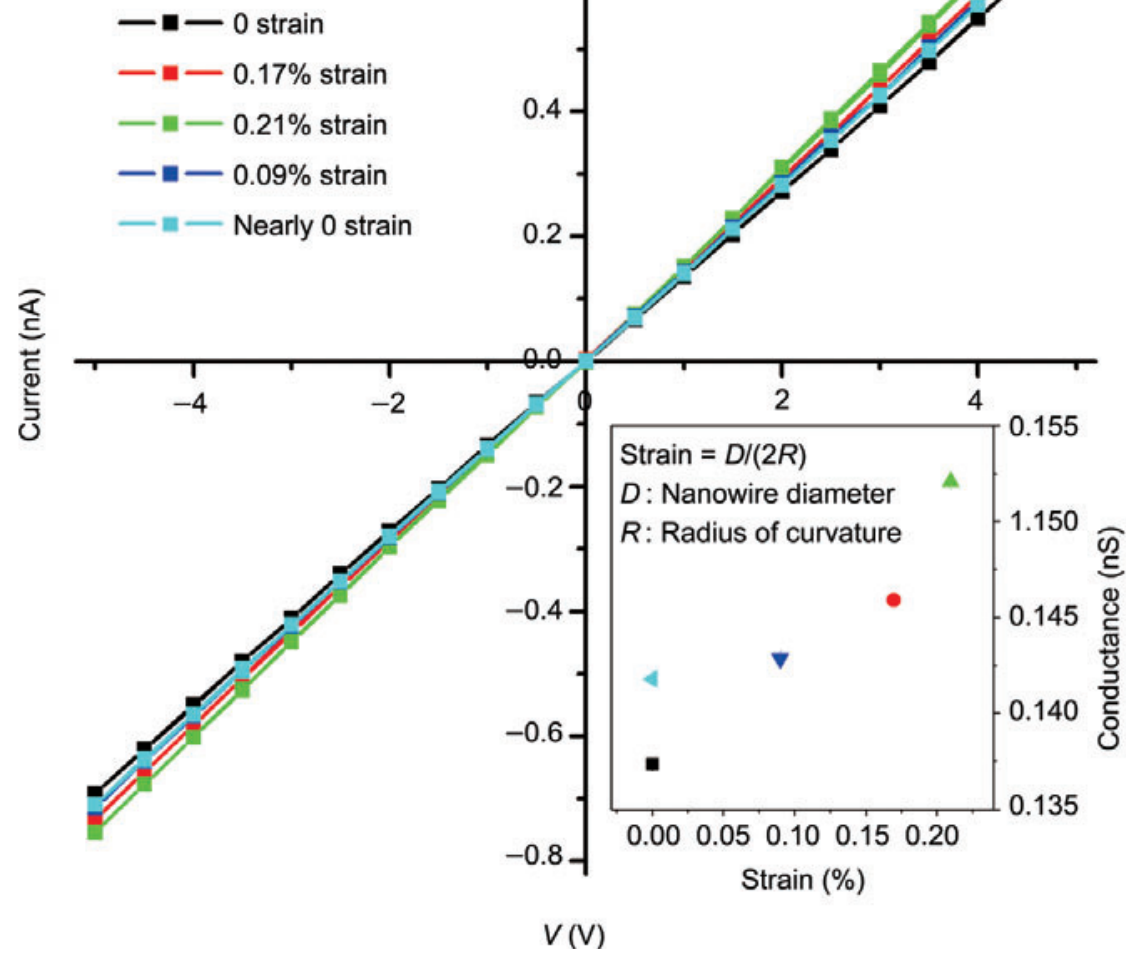

(d)

Figure 3 (a) Schematic illustration of the experimental process; (b) and (c) correspond to images of an individual ZnO NW before bending and being held bent, respectively; (d) $I-V$ relationships of static bending states. The inset shows the variation in conductance with bending strain

bending strain, and the tensile strain may reduce the effective mass in the axial direction. Based on the piezoresistance effect, this effective tensile strainwhich narrows the bandgap-should increase the concentration of lighter carrier in the axial direction and finally enhance the carrier mobility [4] and result in the observed bending-induced conductance enhancement in our electromechanical experiments. As the bending strain in $\mathrm{ZnO} \mathrm{NW}$ itself is quite complicated, the way affects the band structure and enhances the carrier mobility needs further investigation.

In conclusion, we have investigated bendinginduced enhancement in the conductance of individual ZnO NWs or CdS NBs. Different kinds of manipulating tips were used to confirm the validity of the observations. A free moving contact was achieved in order to further confirm the stability of the ohmic contact at the nanowire. These strainsensitive phenomena may find potential applications 
in nanowire-based nanotechnology, flexible electronics and nanoelectromechanical systems.

\section{Acknowledgements}

This work was supported by National Natural Science Foundation of Chian (NSFC) (90606023, 20731160012, 10804003), 973 program (2007CB936202/ 04, 2009CB623703, MOST) of China and NSFC/RGC (N HKUST615/06). D. P. Y. is supported by the Cheung Kong scholar program, and by the Research Fund for the Doctoral Program of Higher Education (RFDP), Ministry of Education, China.

\section{References}

[1] Li, Y.; Meng, G. W.; Zhang, L. D.; Phillip, F. Ordered semiconductor ZnO nanowire arrays and their photoluminescence properties. Appl. Phys. Lett. 2000, 76, 2011-2013.

[2] Kong, Y. C.; Yu, D. P.; Zhang, B.; Fang, W.; Feng, S. Q. Ultraviolet-emitting $\mathrm{ZnO}$ nanowires synthesized by a physical vapor deposition approach. Appl. Phys. Lett. 2001, 78, 407-409.

[3] Huang, M. H.; Mao, S.; Feick, H.; Yan, H.; Wu, Y.; Kind, H.; Weber, E.; Russo, R.; Yang, P. D. Room-temperature ultraviolet nanowire nanolasers. Science 2001, 292, 1897-1899.

[4] He, R.; Yang, P. D. Giant piezoresistance effect in silicon nanowires. Nat. Nanotechnol. 2006, 1, 42-46.
[5] Wang, X.; Zhou, J.; Song, J.; Liu, J.; Xu, N.; Wang, Z. L. Piezoelectric field effect transistor and nanoforce sensor based on a single ZnO nanowire. Nano Lett. 2006, 6, 2768-2772.

[6] Bai, X.; Golberg, D.; Bando, Y.; Zhi, C.; Tang, C.; Mitome, M.; Kurashima, K. Deformation-driven electrical transport of individual boron nitride nanotubes. Nano Lett. 2007, 7, 632-637.

[7] Lin, X.; He, X. B.; Yang, T. Z.; Guo, W.; Shi, D. X.; Gao, H. J.; Ma, D. D. D.; Lee, S. T.; Liu, F.; Xie, X. C. Intrinsic current-voltage properties of nanowires with four-probe scanning tunneling microscopy: A conductance transition of ZnO nanowire. Appl. Phys. Lett. 2006, 89, 043103.

[8] Wang, Z. L.; Song, J. Piezoelectric nanogenerators based on zinc oxide nanowire arrays. Science 2006, 312, 242 246.

[9] Zhou, J.; Gu, Y.; Fei, P.; Mai, W.; Gao, Y.; Yang, R.; Bao, G.; Wang, Z. L. Flexible piezotronic strain sensor. Nano Lett. 2008, 8, 3035-3040.

[10] Zhou, J.; Fei, P.; Gu, Y.; Mai, W.; Gao, Y.; Yang, R.; Bao, G.; Wang, Z. L. Piezoelectric-potential-controlled polarityreversible Schottky diodes and switches of $\mathrm{ZnO}$ wires. Nano Lett. 2008, 8, 3973-3977.

[11] Zhang, Y.; Jia, H. B.; Chen, C. P.; Yu, D. P.; Lee, C. J. Low-temperature growth and Raman scattering study of vertically aligned $\mathrm{ZnO}$ nanowires on Si substrate. Appl. Phys. Lett. 2003, 83, 4631-4633.

[12] Sun, Y.; Thompson, S. E.; Nishida, T. Physics of strain effects in semiconductors and metal-oxidesemiconductor field-effect transistors. J. Appl. Phys. 2007, 101, 104503. 